\title{
FOREWORD
}

\section{Special Section on Fundamentals and Applications of Advanced Semiconductor Devices}

Semiconductor devices and their integrated circuits are key components in recent electronics. For future intelligent and green electronics, new-functional devices featuring high performance and lower power consumption are highly required. This special section on Fundamentals and Applications of Advanced Semiconductor Devices is arranged to discuss fundamentals and applications of semiconductor devices for future advanced electronics. This section contains 8 papers, which cover the fields of process technology and characterization, memory, organic devices, and carbon devices.

I would like to express my thanks to all authors for their contributions to the special section. I also thank all reviewers and editorial committee members for their devoted contribution to reviewing and editing the papers. This special section could not be achieved without their efforts.

Special Section Editorial Committee:

Secretaries: Koji Matsunaga (NEC), Rihito Kuroda (Tohoku Univ.)

Members: Manabu Arai (New JRC), Hiroki Fujishiro (Tokyo Univ. of Science), Masataka Higashiwaki (NICT), Minoru Ida (NTT), Toshihiro Matsuda (Toyama Pref. Univ.), Yasuyuki Miyamoto (Tokyo Inst. of Tech.), Seiichi Miyazaki (Naogya Univ.), Yasushiro Nishioka (Nihon Univ.), Takashi Noguchi (Univ. of the Ryukyu), Shun-ichiro Ohmi (Tokyo Inst. of Tech.), Yutaka Ohno (Nagoya Univ.), Tomoyuki Ohshima (Neophotonics), Hiroshi Okada (Toyohashi Univ. of Tech.), Yukinori Ono (Univ. of Toyama), Taiichi Otsuji (Tohoku Univ.), Naoteru Shigekawa (Osaka City Univ.), Toshihiro Sugii (LEAP), Toshihide Suzuki (Fujitsu Labs.), Shinichi Tanaka (Shibaura Inst. of Tech.), Tadashi Yamaguchi (Renesas)

Naoki Hara, Guest Editor-in-Chief

Naoki Hara (Member)

received the B.E., M.E., and Ph.D. degrees from the University of Tokyo in 1985, 1987, and 1990, respectively. In 1990, he joined Fujitsu Laboratories Ltd., where he has been engaged in the research and development of HEMTs and other heterostructure devices.

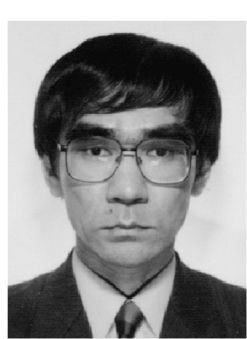

